\title{
Primun non nocere, polypharmacy and pharmacogenetics
}

\section{To the editor}

Primun non nocere, first do no harm, is the oldest precept in medicine. This principle is especially important in polypharmacy, as increasing the number of drugs prescribed can increase the likelihood of adverse events. Personalized medicine offers new approaches to this old problem. Here, we show how the application of a personalized medicine approach helped avoid harm in the management of multiple medications.

A 74-year-old woman with psoriasis was admitted to the cardiology department with congestive heart failure, atrial fibrillation and chronic renal failure. She had a previous history (8 years before) of adverse events with acitretin and cyclosporine. On admission, she was treated with acenocoumarol, bisoprolol, topical clobetasol, clorazepate, digoxin, diltiazem, enoxaparin, furosemide, hydrochlorothiazide, omeprazole and ramipril (Figure 1). Within a few days, she developed erythrodermic psoriasis. After treatment with oral prednisone and topical hydrocortisone, she presented uncontrolled euphoria and disorientation, for which she received alprazolam, which in turn resulted in severe drowsiness and stupor. Worsening erythema on the head, neck and upper chest was treated with methylprednisolone aceponate, which actually increased the severity of the lesions (Figure 1A). Shortly after methotrexate and folic acid were initiated, transaminase elevation and digitalis toxicity were observed.

A personalized pharmacogenetics analysis of theoretical drug-drug interactions [1,2] showed significant compromise of the main steroid-metabolizing enzymes CYP3A4 and CYP3A53 [3] (Figure 1C), among others. We genotyped CYP3A4, CYP3A5, CYP2C9, CYP2C19, CYP2D6 and MDR1. The patient was homozygous for $C Y P 3 A 5^{*} 3$ and heterozygous for $3435 \mathrm{C}>\mathrm{T}$ MDR1. Treatment was readjusted (Figure 1D): omeprazole was replaced with sucralfate; hydrochlorothiazide, enoxaparin, prednisone and methylprednisolone aceponate were suspended; and topical fluocinolone acetonide (nonP450-metabolized steroid derivative) was introduced. Doses of bisoprolol, digoxin and methotrexate were readjusted. The patient improved remarkably with complete remission of cutaneous symptoms (Figure 1B) and control of cardiac, renal and hepatic problems.

There is an increasingly clear understanding of the relationship between genetic variation and drug response [4]. Polypharmacy compromises metabolic pathways, especially in carriers of pharmacogenetic mutations, increasing the risk of adverse effects and consequently increasing costs. Our approach uses personalized drug-drug interaction analysis and pharmacogenotyping to rationalize drug choice and dosing. Although other genetic and nongenetic factors also influence drug response [5], there is an urgent need to translate pharmacogenetics to clinical practice [6]. Our approach has proven useful in recent years.

\footnotetext{
Financial \& competing interests disclosure The authors have no relevant affiliations or financialinvolvement with any organization or entity with a financial interest in or financial conflict with the subject matter or materials discussed in the manuscript. This includes employment, consultancies, honoraria, stock ownership or
}

María Isidoro-García $a^{* 1,2,3}$ Almudena Sánchez-Martín ${ }^{2,4}$, Belén García-Berrocal ${ }^{1,2}$ \& Concepción Román-Curto ${ }^{2,5}$ 'Pharmacogenetics Unit, Department of Clinical Biochemistry, University Hospital of Salamanca, Salamanca, Spain ${ }^{2}$ Biomedical Institute of Salamanca IBSAL, Salamanca, Spain ${ }^{3}$ Department of Medicine, University of Salamanca, Salamanca, Spain ${ }^{4}$ Department of Pharmacy, University Hospital of Salamanca, Salamanca, Spain ${ }^{5}$ Department of Dermatology, University Hospital of Salamanca, Salamanca, Spain *Author for correspondence: Tel.: +34923291373 misidoro@usal.es 

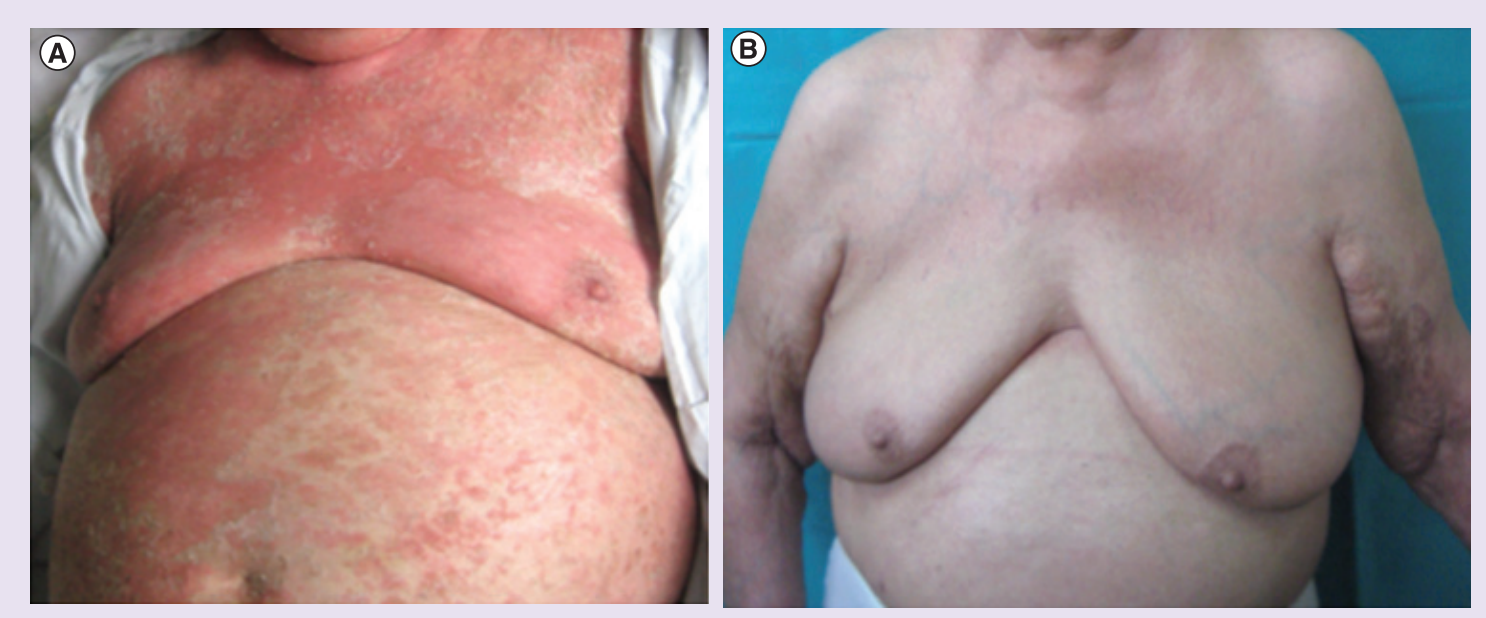

(C)

\begin{tabular}{|c|c|c|c|c|c|c|c|c|c|c|c|c|}
\hline DRUG & DOSE & $1 \mathrm{~A} 1$ & $1 \mathrm{~A} 2$ & 1B1 & $2 \mathrm{C} 8$ & $2 \mathrm{Cg}$ & $2 \mathrm{C} 19$ & 2D6 & $2 \mathrm{E} 1$ & $3 \mathrm{~A} 4$ & $3 A 5$ & $3 \mathrm{~A} 7$ \\
\hline ACENOCOUMAROL & $1 \mathrm{mg} \mathrm{pd}$ & & & & & $\mathrm{IH} / \mathrm{S}$ & & & & & & \\
\hline BISOPROLOL & $5 \mathrm{mg}$ pd & & & & & & & $\mathbf{S}$ & & $\mathbf{S}$ & & \\
\hline CLOBETASOL & Topic & $\mathrm{IH}$ & IH & & & & & & & & & \\
\hline CLORAZEPATE & $5 \mathrm{mg} \mathrm{pd}$ & & & & & & & & & & & \\
\hline DIGOXIN & $0.125 \mathrm{mg} \mathrm{pd}$ & & & & & & & & & $\mathbf{S}$ & & \\
\hline DILTIAZEM & $120 \mathrm{mg}$ pd & & & & $\mathrm{S} / \mathrm{IH}$ & $\mathrm{IH} / \mathrm{S}$ & & $\mathrm{IH} / \mathrm{S}$ & & $\mathrm{IH} / \mathrm{S}$ & $\mathrm{IH} / \mathrm{S}$ & $\mathbf{S}$ \\
\hline ENOXAPARIN & $40 \mathrm{mg} \mathrm{pd}$ & & & & & & & & & & & \\
\hline FUROSEMIDE & $60 \mathrm{mg} \mathrm{pd}$ & & & & & & & & $\mathbf{S}$ & & & \\
\hline HYDROCHLOROTHIAZIDE & $25 \mathrm{mg} \mathrm{pd}$ & & & & & & & & & & & \\
\hline OMEPRAZOLE & $20 \mathrm{mg} \mathrm{pd}$ & $\begin{array}{l}\text { ID/S } \\
\text { /IH }\end{array}$ & $\begin{array}{l}\mathrm{IH} / \\
\text { ID/S }\end{array}$ & ID & $\mathbf{s}$ & $\mathrm{IH} / \mathrm{S}$ & $\begin{array}{l}\text { S/ID } \\
/ / \mathrm{HH}\end{array}$ & IH & & $\begin{array}{c}\mathrm{IH} / \mathrm{S} \\
/ \mathrm{ID}\end{array}$ & $\begin{array}{l}\mathrm{IH} / \mathrm{I} \\
\mathrm{D} / \mathrm{S}\end{array}$ & \\
\hline RAMIPRIL & $5 \mathrm{mg} \mathrm{pd}$ & & & & & & & & & & & \\
\hline PREDNISONE & $0.5 \mathrm{mg} / \mathrm{kg} \mathrm{pd}$ & ID & ID & & & & ID & & & $\begin{array}{c}\text { S/ID } \\
/ / H H\end{array}$ & & \\
\hline HYDROCORTISONE & Topic & & & IH & ID & & & & & $\begin{array}{c}\mathrm{IH} / \mathrm{S} \\
/ \mathrm{ID}\end{array}$ & $\mathbf{S}$ & $\mathbf{S}$ \\
\hline ALPRAZOLAM & & & & & & & & & & $\mathrm{s}$ & $\mathrm{S}$ & $\mathbf{S}$ \\
\hline INSULIN & $100 \mathrm{UI} p d$ & & ID & & & & & & $\mathrm{IH}$ & & & \\
\hline $\begin{array}{l}\text { METHYLPREDNISOLONE } \\
\text { ACEPONATE }\end{array}$ & Topic & & & & & & & & & ID & & \\
\hline METHOTREXATE & $2.5 \mathrm{mg} \mathrm{pw}$ & & & & & & & & & & & \\
\hline FOLIC ACID & $5 \mathrm{mg} \mathrm{pw}$ & & & & & & & & $\mathrm{S}$ & & & \\
\hline
\end{tabular}

(D)

\begin{tabular}{|l|c|c|c|c|c|c|c|c|c|c|c|c|}
\hline \multicolumn{1}{|c|}{ DRUG } & DOSE & $1 \mathrm{~A} 1$ & $1 \mathrm{~A} 2$ & $1 \mathrm{~B} 1$ & $2 \mathrm{C} 8$ & $2 \mathrm{C} 9$ & $2 \mathrm{C} 19$ & $2 \mathrm{D} 6$ & $2 \mathrm{E} 1$ & $3 \mathrm{~A} 4$ & $3 \mathrm{~A} 5$ & $3 \mathrm{~A} 7$ \\
\hline ACENOCOUMAROL & $1 \mathrm{mg} \mathrm{pd}$ & & & & & IH/S & & & & & & \\
\hline BISOPROLOL & $2.5 \mathrm{mg} \mathrm{pd}$ & & & & & & & S & & S & & \\
\hline DIGOXIN & $\begin{array}{c}0.125 \mathrm{mg} \text { pd } \\
(5 \mathrm{~d} \mathrm{pw})\end{array}$ & & & & & & & & & $\mathbf{S}$ & & \\
\hline FUROSEMIDE & $60 \mathrm{mg} \mathrm{pd}$ & & & & & & & & $\mathbf{S}$ & & & \\
\hline RAMIPRIL & $5 \mathrm{mg} \mathrm{pd}$ & & & & & & & & & & & \\
\hline $\begin{array}{l}\text { FLUOCINOLONE } \\
\text { ACETONIDE }\end{array}$ & Topic & & & & & & & & & & & \\
\hline METHOTREXATE & $5 \mathrm{mg} \mathrm{pw}$ & & & & & & & & & & & \\
\hline FOLIC ACID & $5 \mathrm{mg} \mathrm{pw}$ & & & & & & & & $\mathbf{S}$ & & & \\
\hline SUCRALFATE & $1 \mathrm{~g} \mathrm{pd}$ & & & & & & & & & & & \\
\hline
\end{tabular}

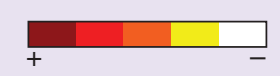

Figure 1. Patient and theoretical drug-drug interaction analysis, before and after the pharmacogenetic management. (A) Patient before pharmacogenetic management. (B) Patient after the treatment adjustment. (C) Theoretical drug-drug interaction analysis for P450 enzymes according to the superCYPplatform, before the treatment adjustment [1]. Enzyme names are shown in the first row (blue). Metabolic pathways from CYP1A1 to CYP3A7 are represented. All drugs prescribed during hospitalization before the adjustments are included in the same analysis. Colors show the degree of compromise. (D) Theoretical drug-drug interaction analysis after the treatment adjustment.

ID: Inductor; IH: Inhibitor; pd: Per day; pw: Per week; S: Substrate. 
options, expert testimony, grants or patents received or pending, or royalties.

\section{References}

1 SuperCYP. A database on cytochrome P450 enzymes. http://bioinformatics.carite.de

2 Pharmgkb. The pharmacogenomics knowledge base. http://pharmgkb.org

3 Kuehl P, Zhang J, Lin Y, Brockmoller J, Hustert E. Sequence diversity in CYP3A promoters and characterization of the genetic basis of polymorphic CYP3A5 expression. Nat. Genet. 27, 383-391 (2001).
The Immunoallergy Association of Salamanca provided funding (ASIAL/01/14) for wrting assistance.

4 Beitelshees AL, Veenstra DL. Evolving research and stakeholder perspectives on pharmacogenomics. JAMA 306, 1252-1253 (2011).

5 Wilkinson GR. Drug metabolism and variability among patients in drug response. N. Engl. J. Med. 352, 2211-2221 (2005).

6 Hamburg MA, Collins FS. The path to personalized medicine. N. Engl. J. Med. 363, 301-304 (2010). 